\title{
Crystal cutters for blocked airways
}

Charcot-Leyden crystals (CLCs) contain the eosinophil granule protein galectin-10 (GAL10) and are a rare example of a protein crystal that occurs spontaneously in vivo. They have long been associated with allergic inflammatory diseases, including severe asthma and chronic rhinosinusitis, yet their biological functions remain unclear. Bart Lambrecht and colleagues have now generated GAL10 crystals that are structurally identical to CLCs and have found that these directly promote type 2 inflammation. Moreover, they have developed antibodies that can 'dissolve' CLCs; these may have therapeutic potential in patients with severe mucus plugging.

In initial screens, Persson et al. showed that GAL10-containing CLCs are abundant in nasal mucosa biopsy samples from patients with severe chronic rhinosinusitis with nasal polyps (CRSwNP), where they strongly correlate with the presence of eosinophil extracellular trap cell death and IL-5 levels. In sputum samples from patients with severe asthma, concentrations of GAL10 also correlated with the extent of eosinophilia. Therefore, as previously reported, CLCs and GAL10 can serve as biomarkers for the presence of eosinophilia.

The authors purified individual CLCs from a patient with CRSwNP and simultaneously generated recombinant dimeric GAL10 as a tagged protein in an in vitro culture system. Removal of the tag caused autocrystallization of the GAL10 preparation into crystals with a CLC-like morphology; crystallography analyses done in collaboration with Savvas Savvides confirmed that the in vitro-generated GAL10 crystals were structurally equivalent to CLCs from the patient. Further analyses identified the crystal-packing interfaces in GAL10, which enabled the authors to generate a mutant form of GAL10 (GAL10 ${ }^{\text {mut }}$ ) that was resistant to autocrystallization.

The authors next injected mice intratracheally with GAL10 crystals, soluble GAL10 ${ }^{\text {mut }}$ or a control vehicle. Mice injected with GAL10 crystals showed rapid infiltration of neutrophils and monocytes into the airways and upregulation of pro-inflammatory cytokines, whereas no notable inflammatory response was detected in mice that received GAL10 ${ }^{\text {mut }}$ or the vehicle. Experiments with fluorescently labelled GAL10 crystals showed that these were taken up by alveolar macrophages in the lungs and also by migratory dendritic cells (DCs). As DCs drive the initiation of adaptive immune responses, the authors tested whether GAL10 crystals can serve as an adjuvant during the induction of antigen-specific immune responses in the airways. Indeed, intratracheal administration of ovalbumin (OVA) mixed with GAL10 crystals (but not OVA and GAL10 ${ }^{\text {mut }}$ ) induced the activation of OVA-specific T cells.

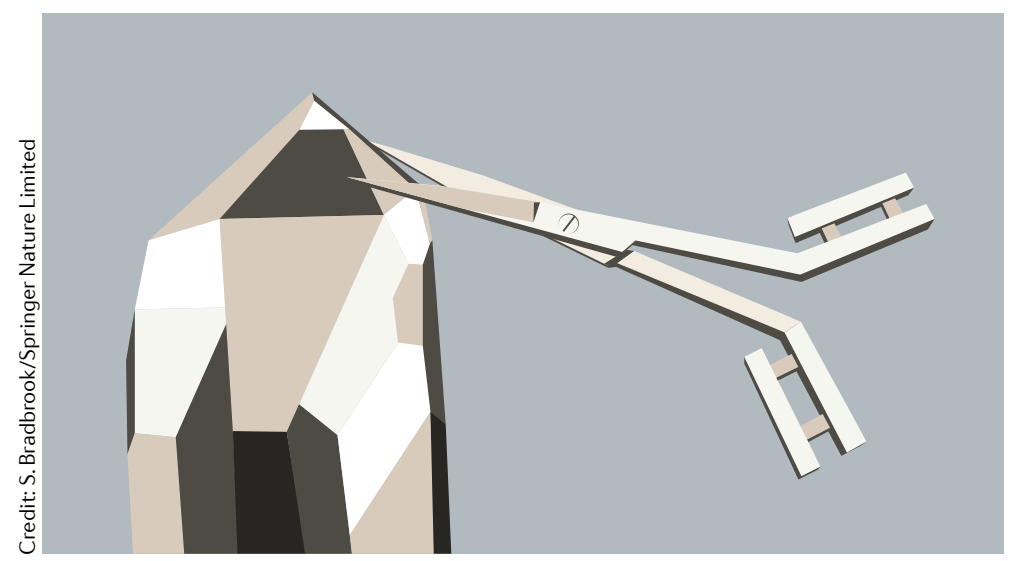

dissolving antibodies targeting GAL10 could be of benefit for relieving mucus

plugging and tissue inflammation in asthma
Furthermore, GAL10 crystals (but not GAL10 ${ }^{\text {mut }}$ ) were able to promote sensitization to OVA and type 2 airway inflammation in a model of allergic airway inflammation.

Previous studies have shown that inorganic and organic crystals can promote inflammation via the NLRP3 inflammasome. However, the GAL10 crystals were able to promote type 2 immunity in mice lacking various inflammasome components. Furthermore, although crystals have been shown to promote inflammation by inducing necroptosis, the authors did not see reduced inflammation when GAL10 crystals were administered to Ripk $3^{--}$mice, which have defective necroptosis.

These findings indicated that targeting inflammasomes or necroptosis is likely to be ineffective in preventing GAL10 crystal-driven inflammation; therefore, the authors adopted a novel approach. They generated several antibodies against the crystal-packing interfaces of GAL10 that were able to block GAL10 autocrystallization in vitro as well as to dissolve preformed GAL10 crystals. Remarkably, the antibodies could dissolve CLCs in fresh mucus samples from patients with CRSwNP and were also protective in a humanized mouse model of CLC-mediated airway inflammation.

These findings indicate that CLCs are not simply a biomarker of tissue eosinophilia in many diseases but are an actual driver of type 2 tissue inflammation and pathology. The authors suggest that their dissolving antibodies targeting GAL10 could be of benefit for relieving mucus plugging and tissue inflammation in asthma and in other severe inflammatory airway diseases. 